\title{
Near-infrared absorbing and luminescent gold speckled silica nanoparticles for photothermal therapy $\dagger$
}

\author{
Parvesh Sharma, ${ }^{a}$ Scott C. Brown, ${ }^{a}$ Amit Singh, ${ }^{a}$ Nobutaka Iwakuma, ${ }^{b}$ Georgios Pyrgiotakis, ${ }^{a}$ Vijay Krishna, ${ }^{a}$ \\ Jacquelyn A. Knapik, ${ }^{c}$ Keira Barr, ${ }^{c}$ Brij M. Moudgil ${ }^{* a}$ and Stephen R. Grobmyer ${ }^{* b}$
}

\author{
Received 8th February 2010, Accepted 28th April 2010 \\ First published as an Advance Article on the web 26th May 2010 \\ DOI: 10.1039/c0jm00354a
}

\begin{abstract}
Herein, we report the development and functional evaluation of sub$50 \mathbf{~ n m}$ near-infrared absorbing and luminescent gold speckled silica (GSS) nanoparticles for fluorescence traceable and selective, in vitro and in vivo photothermal ablation applications. The nanoparticles prepared using microemulsion-mediated approach, are comprised of GRAS (generally regarded as safe) materials, and are promising multifunctional agents for image guided photo-mediated therapies.
\end{abstract}

Engineered nanoparticles exhibiting near-infrared (NIR) absorption are being developed for bio-imaging and cancer therapy by photothermal ablation. ${ }^{1-3}$ Minimal optical absorption by tissue and other biological components in the NIR window (650-900 nm), allowing non-invasive deep tissue light penetration, has led to the development of several photothermal materials e.g. carbon nanotubes (CNT), gold coated CNT, gold (spherical, nanorods, nanocages, agglomerates, hollow, pentagons, large prisms) and composites (silica gold nanoshells, superparamagnetic iron oxide nanoshells, and $\mathrm{Fe}_{3} \mathrm{O}_{4}$-gold nanorods). ${ }^{4-8}$ Cellular and in vivo imaging using these nanomaterials has been accomplished by methods such as reflective confocal microscopy, multi-photon plasmon resonance microscopy, optical coherence microscopy, surface plasmon absorption, computed tomography, magnetic resonance imaging, photoacoustic tomography, surface enhanced Raman spectroscopy etc. ${ }^{5-7,9}$ However, most of these imaging techniques provide limited ability for cellular labeling, imaging, monitoring intracellular localization, tumor penetration, bio-distribution and ex vivo pathological investigations. ${ }^{\mathbf{1 0 , 1 1}}$ Though bio-imaging can be facilitated by integration of widely used tools such as fluorescence, ${ }^{7,9,10}$ the quenching in fluorescence of dyes on direct conjugation to gold nanoparticle surfaces ${ }^{12}$ has remained a major limitation. Additionally, the effective delivery of nanoparticles to cancer sites, as well as deeper penetration into tumors, particularly tumors with small pores, ${ }^{13}$ requires them to be of small size (i.e. all dimensions $<100 \mathrm{~nm}$ ). ${ }^{14-16}$ Overcoming these challenges is necessary for translation of nanoparticles to clinical studies.

Herein we report the development of small (sub-50 nm), NIR absorbing, gold speckled silica (GSS) nanoparticles that retain fluorescence and exhibit therapeutic photothermal properties. The GSS

\footnotetext{
${ }^{a}$ Materials Science and Engineering \& Particle Engineering Research Center, University of Florida, Gainesville, Florida, 32611, USA. E-mail: bmoudgil@perc.ufl.edu; Fax: +352846 1196; Tel: +3528461194

${ }^{b}$ Department of Surgery, University of Florida, Gainesville, Florida, 32610, USA. E-mail: Stephen.grobmyer@surgery.ufl.edu; Tel: +352 2650169

'Department of Pathology, University of Florida, Gainesville, Florida, USA $\dagger$ Electronic supplementary information (ESI) available: Additional experimental details. See DOI: 10.1039/c0jm00354a
}

nanoparticles have a silica core with discontinuous, irregular gold nanodomains on the surface and within the pores of the silica matrix. The dielectric-metal interfaces enable the particles to absorb a broad spectrum of light from the visible to NIR regions - facilitating their use as photothermal ablation agents. Integration of a fluorescent agent within the silica core makes GSS nanoparticles readily
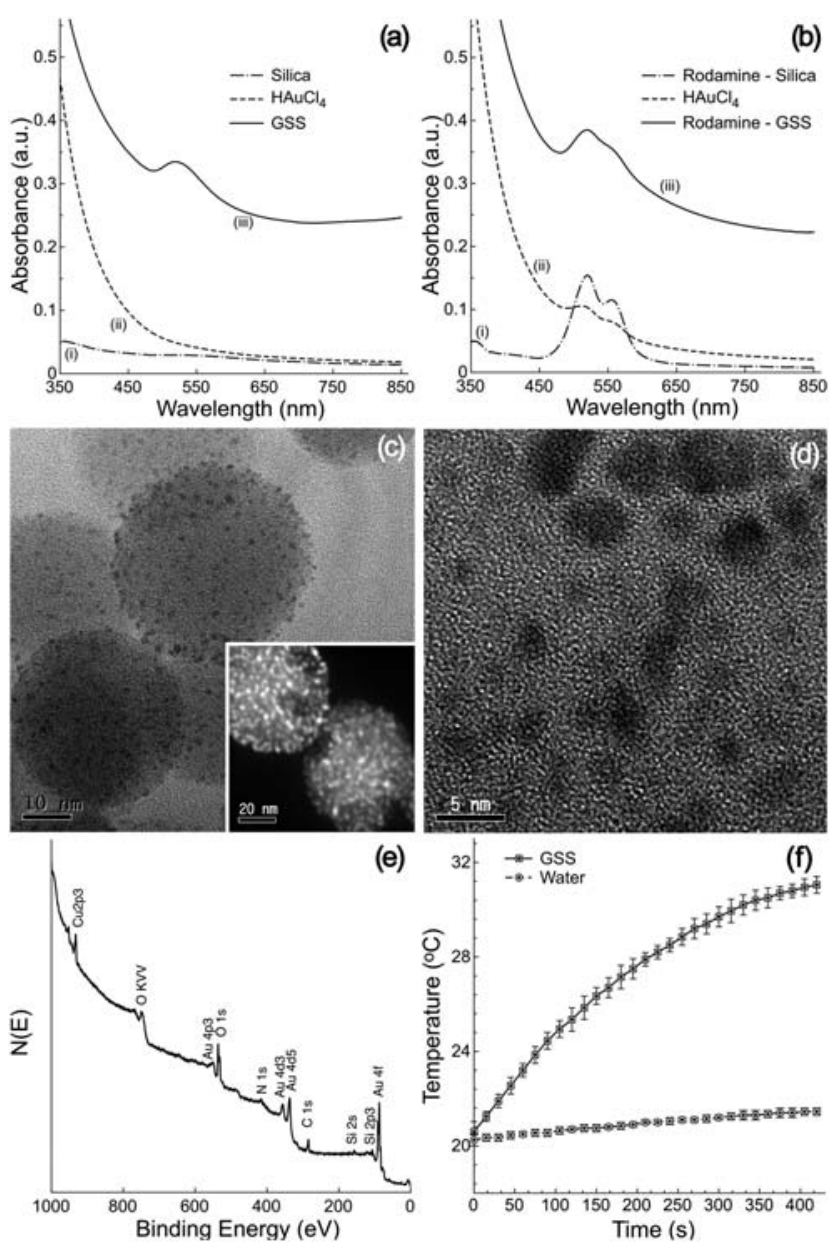

Fig. 1 The absorbance spectra of the microemulsion at different stages of the synthesis for (a) GSS nanoparticles: (i) silica nanoparticles; (ii) after $\mathrm{HAuCl}_{4}$ addition and (iii) GSS nanoparticles; (b) rhodamine doped GSS nanoparticles; (c) representative TEM of $c a .40 \mathrm{~nm}$ GSS nanoparticles showing the speckled silica surface; inset z-contrast digital TEM; (d) higher magnification showing irregular shaped, discontinuously placed, 1-5 nm crystalline nano-gold deposits on silica; (e) representative XPS spectra and (f) $c a .11^{\circ} \mathrm{C}$ increase in temperature of pegylated GSS nanoparticles in water suspension. 
imageable for high throughput in vitro fluorescence assays and ex vivo histopathological analyses. Additionally, silica and gold surfaces allow bioconjugation for targeting applications. Both silica and gold are generally regarded as safe (GRAS) biomaterials, and gold-silica nanoshells are currently undergoing early phase clinical trials. ${ }^{17}$ Because of their multifunctionality, GSS nanoparticles hold the potential for use in image guided therapies. The synthesis and characterization of fluorescently labeled GSS nanoparticles, their uptake in breast and lung cancer cells, intracellular localization, NIR absorbance and photothermal ablation functional evaluations (in vitro and in vivo) are presented.

GSS nanoparticles were synthesized in one pot using a water-in-oil (w/o) microemulsion. The use of a microemulsion-based approach differs from other methods reported for fabricating dielectric-gold core-shell nanostructures (e.g., sol-gel ${ }^{18}$ and layer-by-layer deposition. ${ }^{19}$ ) In w/o microemulsions, the manipulation of the water to surfactant molar ratio $\left(\mathrm{W}_{\mathrm{o}}\right)$ provides the ability to alter particle size and presents a confined surfactant stabilized aqueous environment to engineer multiple modalities in nanoparticles. ${ }^{20} \mathrm{GSS}$ nanoparticles were prepared using a modified w/o microemulsion protocol ${ }^{21}$ comprising of Tx-100, cyclohexane, n-hexanol (1:4.2:1 v/v). The progress of the reaction for non-fluorescent GSS nanoparticles at each step of synthesis was monitored by UV-vis absorption spectroscopy (Fig. 1a). Briefly, the silica core was first formed by the hydrolysis and co-condensation of tetraethyl orthosilicate (TEOS) and aminopropyl triethoxysilane (APTS) precursors encapsulated in the microemulsion (Fig. 1a(i)). The presence of the APTS during the silica nanoparticle formation and growth stage introduces free amine groups which provide adhesion sites for nanogold deposits in subsequent steps. The fluorescent silica core was similarly prepared using covalently coupled fluorescein isothiocyanate (FITC), or rhodamine APTS conjugate ${ }^{22}$ as a precursor. Fig. 1a(ii) and (iii) show the extinction spectra after the $\mathrm{HAuCl}_{4}$ addition to the microemulsion containing silica nanoparticles and subsequent reduction with a dilute solution of reducing agent. Fig. 1a(iii) shows the surface plasmon peak at $\sim 530 \mathrm{~nm}$ and a broad, continuous extinction spectra of the GSS nanoparticles extending into the NIR region indicating the deposition of gold on silica. The extinction spectra of rhodamine doped GSS nanoparticles (Fig. 1b) follows a similar trend to the undoped GSS nanoparticles with the overlapping regions of absorption maxima for rhodamine and gold nanodomains. As compared to the core silica nanoparticles and similarly sized gold nanoparticles ${ }^{23}$ which have minimal absorption and scattering, the GSS nanoparticles show an increase of absorption in the $600-850 \mathrm{~nm}$ range. Distinct from the nanoshell synthesis, in which $2 \mathrm{~nm}$ gold nanoparticle seeds are used to grow a shell on silica nanoparticles (prepared separately), the gold was generated and deposited in situ (in microemulsions) within and on the silica nanoparticle matrix. This method generates 1-5 nm, irregular shaped, crystalline, discontinuous deposits of gold on silica nanoparticles. The surfactant layers capping the as-formed GSS nanoparticle allow controlled and slow reduction into sterically stabilized non-aggregated particles. In GSS synthesis, we have found that aggregation is dependent on the amount of gold used in the reaction and can cause microemulsion instability at high concentrations.

GSS nanoparticles offer a combination of three distinct features: size tunability (by changing the $\mathrm{W}_{\mathrm{o}}{ }^{24}$ ), fluorescence, and visible-NIR broad extinction spectra. Fig. 1c shows the TEM of narrowly monodispersed, sub-50 nm GSS nanoparticles and inset shows the z-contrast
TEM. The discontinuous and random deposits of nanogold on silica surface (Fig. 1d) enable visible to NIR absorbance as well as the retention of fluorescence. This is in contrast to other materials such as gold nanoshells, nanocages and nanorods that exhibit NIR absorbance but not the fluorescence due to quenching of fluorescence by gold surface plasmon interactions. ${ }^{12}$ Jin et al. ${ }^{25}$ have recently reported combination of fluorescent (quantum dot) and plasmonic (gold) properties in a single nanoparticle by controlling the spacing using a layer-by-layer assembly approach. The GSS nanoparticles similarly remain brightly fluorescent due to the ability of light to penetrate the silica surface, and separation of the fluorescent dyes in a silica matrix from nanogold speckles on the surface..$^{25}$ Gold speckled surface of GSS nanoparticles is also responsible for the extinction maximum at $530 \mathrm{~nm}$ and broad extinction extending into the NIR region (Fig. 1a). In comparison, $40 \mathrm{~nm}$ gold nanoshells ${ }^{26}$ are shown to have a maximum extinction peak at $644 \mathrm{~nm}$ and a broad extinction spectrum. The authors also report that these small nanoshells are prone to aggregation. Ji et al. have similarly reported a broad extinction spectra between $700-900 \mathrm{~nm}$ for $80 \mathrm{~nm}$ gold nanoshells. ${ }^{27}$ The representative X-ray photoelectron spectroscopy (XPS) spectrum of GSS nanoparticles (Fig. 1e) shows the characteristic peaks for gold and silica in the hybrid particle. The silica to gold mass ratio for NIR absorbing GSS nanoparticles (determined by Inductively Coupled Plasma Spectroscopy) was found to be in range of 0.41 to 0.55 . The ability of GSS nanoparticles to generate heat was tested by measuring the temperature increase of the GSS nanoparticles suspension upon illumination with NIR light. Using a $785 \mathrm{~nm}$ CW NIR laser (output $350 \mathrm{~mA} ; 500 \mathrm{~mW}$ ) incident on cuvette containing $1 \mathrm{ml}$ (of $5 \mathrm{mg} / \mathrm{mL}$ ) pegylated GSS nanoparticles for $5 \mathrm{~min}$, a rise in temperature of nearly $11^{\circ} \mathrm{C}(\mathrm{Std}$. dev. \pm 1 ) was observed (Fig. 1f).

The fluorescence and photothermal ability of the fluorescently doped GSS nanoparticles has been demonstrated in vitro with the lung A549 cancer cell line (detailed procedure in ESI $\dagger$ ). The green fluorescence from the FITC doped GSS nanoparticles allows visualization of the nanoparticles inside the cells, and further shows their presence in the perinuclear region of the cancer cell (nuclei stained with the blue dye) (Fig. 2a). This can be observed conclusively in Fig. $2 b$ which shows the z-stacked cross sectional image of the GSS labeled cells. The ability of the GSS nanoparticles to traffic to the perinuclear region can be an important tool for cellular therapy. In separate experiments, the GSS nanoparticle uptake by human breast cancer cells was analyzed quantitatively as a function of particle dosage by flow cytometry and a dose associated uptake of GSS was observed by the cells (ESI $\dagger$ ). These experiments demonstrate the ability to perform standard fluorescence based laboratory procedures with GSS nanoparticles to estimate particle uptake/dosimetry as well as monitor intracellular localization. The in vitro photothermal ablation of human A549 lung carcinoma cells was done using the NIR laser (785nm) present on the Renishaw InVia Confocal Raman Instrument which does not cause any damage to the cells even under prolonged exposure times. ${ }^{28}$ The FITC doped GSS nanoparticle labeled cells were exposed to the NIR laser along a linear path followed by a cell viability test with trypan blue. Cells exposed to high temperatures can undergo a rapid necrotic death resulting in membrane permeation. Fig. $2 \mathrm{c}$ and d (magnification) show the dead cells as stained by trypan blue along the path of the laser, with minimal collateral damage to surrounding cancer cells. It indicates that the localized rise in the temperature of GSS particles leads to rapid injury and death of the labeled cells. 

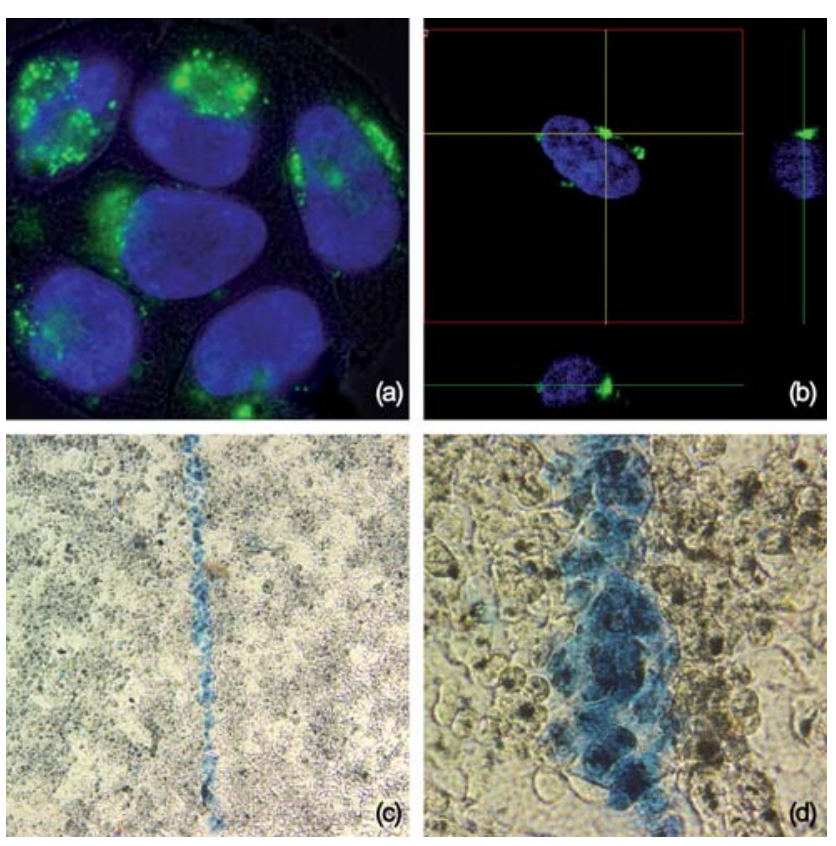

Fig. 2 (a) Representative confocal microscope picture of lung A549 cells labeled with the FITC doped GSS nanoparticles showing the presence of nanoparticles (green) near the nucleus (blue — stained with Hoechst). (b) A z-position cross section showing the localization of GSS nanoparticles adjacent to the nuclear boundary. (c) Trypan blue stained dead cells as ablated selectively along the path of the NIR laser and unharmed surrounding cells. (d) Higher magnification of trypan blue stained dead cells.

The ability of nanoparticles to induce photothermal ablation in vivo is challenging because of issues such as absorbance and scattering by tissue, dispersion state of particles, depth and spread of particles, and the wavelength of laser employed. To evaluate the performance ability of the GSS nanoparticles to mediate thermal ablation in vivo, GSS nanoparticles (300 $\mu \mathrm{g}$ in saline) or saline controls were injected intratumorally into nu/nu mice bearing human breast cancers. The tumor region was irradiated with NIR laser (output $350 \mathrm{~mA} ; 500 \mathrm{~mW}$ ) for $10 \mathrm{~min}$. Fig. $3 \mathrm{~b}$ shows $\mathrm{ca}$. $60 \%$ necrosis in tumors with GSS nanoparticles and NIR ablation as compared to ca. $30 \%$ necrosis in control experiments $(\mathrm{p}=0.001)$. It should be noted that the BT474 xenograft tumor model applied in the current research develops a central region of necrosis during tumor progression. ${ }^{29,30}$ Microscopic examination of the untreated BT474

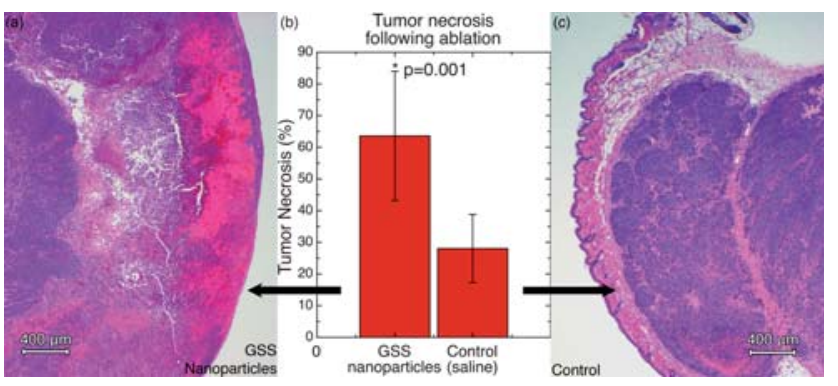

Fig. 3 Representative H \& E stained histological image of ex vivo tumor sections of photo-thermally ablated tumors injected with: (a) GSS nanoparticles, (c) saline injection; (b) significantly more necrosis is observed in tumors treated with GSS compared to control $(\mathrm{n}=5)$. tumors by hematoxylin and eosin $(\mathrm{H} \& \mathrm{E})$ staining revealed nodular masses composed of large epithelioid cells with pleomorphic nuclei, prominent nucleoli, and numerous atypical mitotic figures. Individual necrotic cells as well as areas of frank necrosis were present within the centers of the majority of the tumor nodules (not shown). Areas with individual cell necrosis and impending frank necrosis were significantly increased upon tumor ablation with GSS nanoparticles (Fig. 3a) as compared to controls (Fig. 3c). Ongoing work is focused on optimization of delivery of these multifunctional nanoparticles to tumor sites and determinates of minimal dosing to achieve in vivo photothermal therapy.

\section{Conclusions}

In summary, we present the development and functional evaluation of sub-50 nm NIR absorbing and luminescent GSS nanoparticles for fluorescence traceable and selective, in vitro and in vivo photothermal ablation applications. Prepared in one pot, using microemulsions, gold is generated and deposited in situ on fluorescently doped silica nanoparticles as $1-5 \mathrm{~nm}$, irregular shaped, randomly-present nanodomains. The discontinuous gold deposits, as opposed to a continuous gold film, are hypothesized to lead to broad NIR absorption characteristic of the GSS nanoparticles and also prevent quenching of the fluorescent dye. The fluorescent particles provide a facile and widely applicable means to monitor cancer cell uptake, internalization, localization, tumor penetration and bio-distribution and their small size would allow them to extravasate to tumor sites. Doping with NIR fluorescent dyes will permit the real time non-invasive imaging and therapy with GSS nanoparticles. Further, the GRAS composition of GSS is favorable for future clinical and theranostics applications for the treatment of cancer.

\section{Acknowledgements}

PERC, the National Science Foundation (NSF Grants EEC-9402989, EEC-0506560), Bankhead Cooley, James \& Esther King Biomedical Research Program (Grant 06NIR-05) and Patricia Adams Cancer Nanotechnology Research Fund, are acknowledged for support of this research. The authors thank Kerry Siebein for assistance with HRTEM.

\section{Notes and references}

1 P. K. Jain, X. H. Huang, I. H. El-Sayed and M. A. El-Sayed, Acc. Chem. Res., 2008, 41, 1578-1586.

2 M. Hu, J. Y. Chen, Z. Y. Li, L. Au, G. V. Hartland, X. D. Li, M. Marquez and Y. N. Xia, Chem. Soc. Rev., 2006, 35, 1084-1094.

3 C. Loo, A. Lin, L. Hirsch, M. H. Lee, J. Barton, N. Halas, J. West and R. Drezek, Technol. Cancer Res. Treat., 2004, 3, 33-40.

4 J. Kang, J. Yang, J. Lee, S. J. Oh, S. Moon, H. J. Lee, S. C. Lee, J. H. Son, D. Kim, K. Lee, J. S. Suh, Y. M. Huh and S. Haam, J. Mater. Chem., 2009, 19, 2902-2905.

5 J. W. Kim, E. I. Galanzha, E. V. Shashkov, H. M. Moon and V. P. Zharov, Nat. Nanotechnol., 2009, 4, 688-694.

6 G. V. C. Maltzahn, A. J. Park, R. Ramanathan, M. J. Sailor, T. A. Hatton and S. N. Bhatia, Adv. Mater., 2009, 21, 3175-3180.

7 W. Lu, C. Y. Xiong, G. D. Zhang, Q. Huang, R. Zhang, J. Z. Zhang and C. Li, Clin. Cancer Res., 2009, 15, 876-886.

8 S. Lal, S. E. Clare and N. J. Halas, Acc. Chem. Res., 2008, 41, 1842 1851.

9 M. P. Melancon, W. Lu, Z. Yang, R. Zhang, Z. Cheng, A. M. Elliot, J. Stafford, T. Olson, J. Z. Zhang and C. Li, Mol. Cancer Ther., 2008, 7, 1730-1739. 
10 X. Michalet, F. F. Pinaud, L. A. Bentolila, J. M. Tsay, S. Doose, J. J. Li, G. Sundaresan, A. M. Wu, S. S. Gambhir and S. Weiss, Science, 2005, 307, 538-544.

11 L. L. Yang, H. Mao, Y. A. Wang, Z. H. Cao, X. H. Peng, X. X. Wang, H. W. Duan, C. C. Ni, Q. G. Yuan, G. Adams, M. Q. Smith, W. C. Wood, X. H. Gao and S. M. Nie, Small, 2009, 5, 235-243.

12 F. Cannone, G. Chirico, A. R. Bizzarri and S. Cannistraro, J. Phys. Chem. B, 2006, 110, 16491-16498.

13 G. Kong, R. D. Braun and M. W. Dewhirst, Cancer Res., 2000, 60, 4440-4445.

14 X. M. Qian, X. H. Peng, D. O. Ansari, Q. Yin-Goen, G. Z. Chen, D. M. Shin, L. Yang, A. N. Young, M. D. Wang and S. M. Nie, Nat. Biotechnol., 2008, 26, 83-90.

15 A. G. Cuenca, H. B. Jiang, S. N. Hochwald, M. Delano, W. G. Cance and S. R. Grobmyer, Cancer, 2006, 107, 459-466.

16 S. D. Perrault, C. Walkey, T. Jennings, H. C. Fischer and W. C. W. Chan, Nano Lett., 2009, 9, 1909-1915.

17 J. R. Cole, N. A. Mirin, M. W. Knight, G. P. Goodrich and N. J. Halas, J. Phys. Chem., 2009, 113, 12090-12094.

18 S. J. Oldenburg, R. D. Averitt, S. L. Westcott and N. J. Halas, Chem. Phys. Lett., 1998, 288, 243-247.

19 F. Caruso, Adv. Mater., 2001, 13, 11.

20 P. Sharma, S. Brown, M. Varshney and B. Moudgil, in Interfacial Processes and Molecular Aggregation of Surfactants, ed. R. Narayanan, Springer, 2008, pp. 189-233.
21 P. Sharma, S. C. Brown, N. Bengtsson, Q. Z. Zhang, G. A. Walter, S. R. Grobmyer, S. Santra, H. B. Jiang, E. W. Scott and B. M. Moudgil, Chem. Mater., 2008, 20, 6087-6094.

22 S. Santra, H. Yang, D. Dutta, J. T. Stanley, P. H. Holloway, W. H. Tan, B. M. Moudgil and R. A. Mericle, Chem. Commun., 2004, 2810-2811.

23 P. K. Jain, K. S. Lee, I. H. El-Sayed and M. A. El-Sayed, J. Phys. Chem. B, 2006, 110, 7238-7248.

24 P. Sharrna, S. Brown, G. Walter, S. Santra and B. Moudgil, $A d v$. Colloid Interface Sci., 2006, 123-126, 471-485.

25 Y. D. Jin and X. H. Gao, Nat. Nanotechnol., 2009, 4, 571-576.

26 M. R. Rasch, K. V. Sokolov and B. A. Korgel, Langmuir, 2009, 25, $11777-11785$.

27 X. J. Ji, R. P. Shao, A. M. Elliott, R. J. Stafford, E. Esparza-Coss, J. A. Bankson, G. Liang, Z. P. Luo, K. Park, J. T. Markert and C. Li, J. Phys. Chem. C, 2007, 111, 6245-6251.

28 I. Notingher, S. Verrier, H. Romanska, A. E. Bishop, J. M. Polak and L. L. Hench, Spectrosc.-Int. J., 2002, 16, 43-51.

29 H. J. van Slooten, B. A. Bonsing, A. J. Hiller, G. T. Colbern, J. H. van Dierendonck, C. J. Cornelisse and H. S. Smith, Br. J. Cancer, 1995, 72, 22-30.

30 K. L. Li, L. J. Wilmes, R. G. Henry, M. G. Pallavicini, J. W. Park, D. D. Hu-Lowe, T. M. McShane, D. R. Shalinsky, Y. J. Fu, R. C. Brasch and N. M. Hylton, J. Magn. Reson. Imaging, 2005, 22, 511-519. 